\title{
基于苯并噻唑的新型双嗍桥联梯形共轭骨架的合成、结构及其性质
}

\author{
张振宇 ${ }^{a}$ 李婉君 ${ }^{b}$ 叶开其* ${ }^{*}$ 张红雨*, $a$ \\ ( ${ }^{a}$ 吉林大学化学学院超分子结构与材料国家重点实验室＼cjkstart长春 130012) \\ $\left({ }^{b}\right.$ 太原工业学院化学与化工系 太原 030008)
}

\begin{abstract}
摘要 设计合成了一个新型双嗍桥联梯形分子. 该有机分子拥有一个拓展的 $\pi$ 共轭骨架结构. 通过真空升华方法, 得 到了这个化合物的单晶. 单晶 X 射线衍射分析表明该化合物拥有一个完全共平面的并七环梯形骨架. 与每一个嗍配位 的米基基团可以有效地隔离发光单元，避免聚集诱导淬灭. 化合物具有非常高的熔点和热分解温度，表明其拥有良好 的热稳定性. 电化学、光物理性质和理论计算研究表明, 我们设计的双硼梯形共轭化合物在有机电子发光二级管器件 中具有潜在的应用价值. 因此, 构筑了以该分子为发光层和电子传输层的器件, 得到了不错的电致发光效果.
\end{abstract}

关键词 四配位硼; 梯形结构; $\pi$ 共轭分子; 有机电致发光二极管; 苂光发光体

\section{Synthesis, Structure and Properties of a Novel Benzothiazole-based Diboron-Bridged $\pi$-Conjugated Ladder}

\author{
Zhang, Zhenyu $^{a} \quad$ Li, Wanjun $^{b} \quad$ Ye, Kaiqi*,a $\quad$ Zhang, Hongyu ${ }^{*, a}$ \\ ( ${ }^{a}$ State Key Laboratory of Supramolecular Structure and Materials, College of Chemistry, Jilin University, Changchun \\ 130012) \\ ( ${ }^{b}$ Department of Chemistry and Chemical Engineering, Taiyuan Institute of Technology, Taiyuan 030008)
}

\begin{abstract}
A novel diboron-bridged ladder-type molecule with extended $\pi$-conjugated skeleton has been designed and synthesized. Single crystal of the compound has been grown by the method of vacuum sublimation and the molecular structure determined by X-ray diffraction analysis demonstrate that this ladder-type molecule has a seven-ring fused skeleton, which is almost coplanar. And the two mesityl groups coordinated to each boron atom can effectively keep the luminescent units apart. No $\pi-\pi$ interaction can be observed between the two extended $\pi$-conjugated planes. In the packing structures, we cannot find the intramolecular hydrogen bond, $\mathrm{C}-\mathrm{H} \cdots \pi$ interaction and other weak interaction. Based on UV-vis absorption and fluorescence emission spectra, the longest absorption band is peaked at $372 \mathrm{~nm}$ in dichloromethane solution and the emission band is at $544 \mathrm{~nm}$ which has a large stokes shift of $8499 \mathrm{~cm}^{-1}$. In the solid state, the compound shows yellow fluorescence with emission peak at $582 \mathrm{~nm}$. The compound in condensed phase displays only slightly red shifted emission spectra and almost the same fluorescence quantum yield compared to that in dispersed phase, which is attributed to the bulky side groups on the boron atoms. The compound possesses a very high melting point $\left(T_{\mathrm{m}}=352{ }^{\circ} \mathrm{C}\right)$ and decomposition temperature $\left(T_{\mathrm{d} 5}=\right.$ $360{ }^{\circ} \mathrm{C}$ ) due to the rigid $\pi$-conjugated plane that indicates its good thermal stability. The compound has two pairs of reversible reduction peaks and an irreversible oxidation peak which are similar to the reported four-coordinate compounds. The cyclic voltammogram curves indicate boron chelation can greatly lower the lowest unoccupied molecular orbital (LUMO). Thus, it makes cathodic reductions easier and thereby endows the $\pi$-conjugated ladder with enhanced electron-accepting nature. The electrochemical property suggests that the compound is suitable as an electron-transporting layer in organic light-emitting diode (OLED) devices. To obtain a deeper insight into the electronic structure and energy levels of the $\pi$-conjugated skeleton, density functional theory (DFT) calculations were performed. The LUMOs are delocalized on the entire seven-ring $\pi$-conjugated ladder while the central benzene ring and the mesityl chelated with the boron atom make a main contribution to the HOMOs. The general trend of the calculated HOMO/LUMO levels and energy gaps are basically consistent with the electrochemical and the photophysical data. Therefore, we fabricated OLEDs devices using the molecule as emitter and/or electron-transporting layers, which showed good EL performance.

Keywords four-coordinate boron; ladder-type skeleton; $\pi$-conjugated molecule; OLEDs; fluorescence emitter
\end{abstract}

\section{1 引言}

在有机电子学领域, 梯形共轭分子吸引了越来越多
的关注. 梯形共轭有机分子, 即所有的环并在一起的共 轭骨架, 拥有很重要的应用潜能 ${ }^{[1]}$. 将主族元素, 如: 嗍、硅、磷、硫、硒等，掺入梯形共轭骨架中作为桥联

* E-mail: hongyuzhang@jlu.edu.cn

Received September 14, 2015; published September 21, 2015.

Supporting information for this article is available free of charge via the Internet at http://sioc-journal.cn.

Project supported by the National Natural Science Foundation of China (No. 51173067).

项目受国家自然科学基金(No. 51173067)资助. 
基团是一种修饰梯形共轭有机分子的有效策略.

嗍原子是一个缺电子的原子, 其价电子结构为 $2 s^{2} 2 p^{1}$, 可采用 $\mathrm{sp} 2$ 杂化形成缺电子的三配位硼化合 物 $^{[2]}$. 三配位硼化合物在有机合成等领域得到了深入研 究. 由于三配位硼具有空的 $\mathrm{p}$ 轨道, 可以进一步与路易 斯碱反应得到四配位硼化合物. 有机四配位硼化合物由 于其独特而优异的性质被广泛应用于有机电致发光二 极管 $(\mathrm{OLEDs})^{[3]}$ 、有机太阳能电池 $(\mathrm{OPVs})^{[4]}$ 、有机固体激

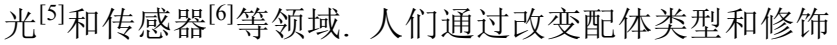
嗍原子上取代基等方法设计合成了大量的四配位路化 合物. 其中, 比较常见的是 $\mathrm{B}$ 原子与 $\mathrm{N}^{\wedge} \mathrm{O}^{[7]}, \mathrm{N}^{\wedge} \mathrm{N}^{[8]}$ 和 $\mathrm{N}^{\wedge} \mathrm{C}^{[9]}$ 螯合的体系. 将四配位嗍化合物与梯形共轭 体系结合在一起, 形成分子中包含多个硼原子的刚性共 轭化合物的报道还是比较少见的. 近几年, 我们课题组 分别报道了以噻唑并噻唑衍生物和双苯并噻唑衍生物 为配体的双硼桥联梯形分子的合成. 将这些分子作为电 子传输层和苂光发光层应用于有机电致发光二极管器 件中, 取得了很好效果 ${ }^{[10]}$. 日本名古屋大学的 Yamaguchi 教授课题组 ${ }^{[11]}$ 一直致力于主族元素掺杂的梯 形共轭分子的研究, 他们合成了嗍和其他原子掺杂的梯 形共轭分子具有比较有趣的光物理化学性质. Curiel 等 ${ }^{[12]}$ 报道了一系列基于吲哚并咔唑配体的梯形硼化合 物，表现出了优异的发光性能.

梯形嘲共轭分子具有很强的固体发光效率和很高 的载流子迁移率, 利用它们作为发光材料或者是电子传 输材料来构筑结构简单的有机电致发光器件表现出了 很好的电致发光效率. 在梯形结构分子中，硼原子被引 入到共轭骨架中, 通过限制配体共轭骨架的振动, 从而 减少振动非辐射跃迁引起的能量损失来提高分子的发 光效率. 并且硼原子的引入还能够通过降低分子的最低 非占有轨道(LUMO) 能级来调节分子的电子能态, 增加 分子的电子亲和能力. 虽然梯形嗍共轭分子有如此多的 优点, 但是, 到目前为止, 由于缺乏有效的合成方法, 含有多个硼原子的共轭梯形分子的报道还非常少.

最近, 我们已经开发了一种简单的方法来合成硼原 子掺杂的梯形共轭体系. 基于这样的方法, 我们已经合 成了一系列高发光效率的有机梯形嗍共轭分子. 它们在 有机电致发光二极管器件中表现出了很好的效果. 在本 文中, 我们利用一个苯环对位连接两个苯并噻唑这样一 个简单的有机分子作为构筑单元, 将带有两个米基基团 的四配位嗍引入到梯形体系中构筑刚性的梯形共轭骨 架. 得到的化合物具有七环共平面的梯形共轭结构. 米 基基团的引入可以有效地减小平面骨架的分子间相互 作用，特别是 $\pi-\pi$ 相互作用，从而有利于提高材料在聚 集态下的发光效率. 我们对这样一个化合物的晶体结 构、紫外-可见吸收光谱、苂光发射光谱、热力学稳定 性和电化学稳定性进行了表征和测试, 并对化合物分子 进行了理论计算. 测试结果表明: 该化合物在有机电致
发光二极管器件中有很大的应用潜能. 我们利用该分子 作为发光层和电子传输层构筑电致发光器件, 得到了较 好的电致发光效率.

\section{2 结果与讨论}

\section{1 合成}

梯形硼共轭分子 2 的合成如图 1 所示. 将得到的配 体化合物 1 利用正丁基锂活化之后，滴入二米基氟化硼 的四氢呋喃溶液，室温搅拌过夜. 蒸干溶剂后，粗产品 经过真空梯度升华即可得到目标分子 $\mathbf{2}$ 的纯品. 为了确 定其结构和纯度，合成的化合物 2 分别经过核磁共振波 谱、质谱、元素分析和单晶 $\mathrm{X}$ 射线衍射进行了详细的表 征.

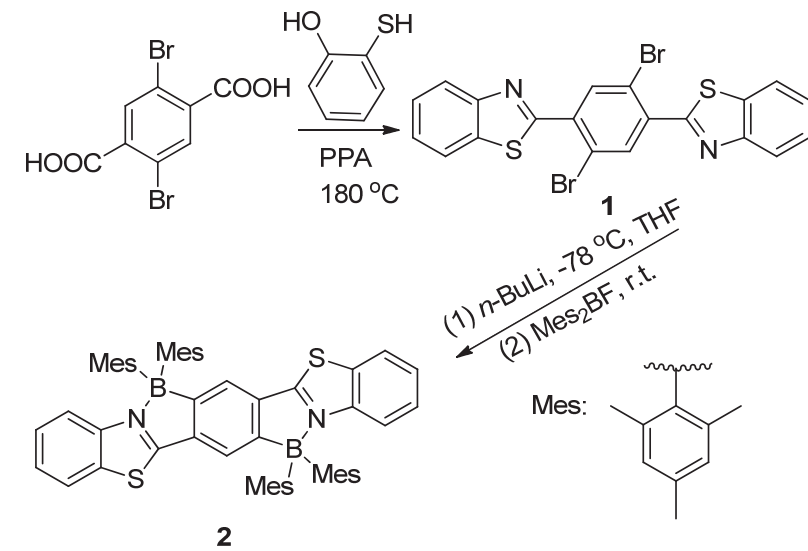

图 1 梯形嗍共轭分子的合成路线图

Figure 1 Synthetic procedure of the diboron ladder-type $\pi$-conjugated molecule

\section{2 晶体结构}

为了更直观地理解和认识化合物 2 的构型以及分子 堆积结构，我们用真空梯度升华的方法得到了梯形喼共 轭分子的晶体. 晶体为橙黄色、块状，属于单斜晶系，为 $P 2(1) / n$ 空间群. 从图 2 左图我们可以看到, 从配体 1 到 梯形硼分子 2 , 确实形成了新的 $\mathrm{B}-\mathrm{C}, \mathrm{B}-\mathrm{N}$ 键, 其键长 分别为 1.64 和 $1.67 \AA$. 这样的键长与之前文献报道的典 型的四配位硼配位键非常接近. 喼原子通过与碳原子和 氮原子的配位作用螯合形成的一个新的五元环。这样的 五元环使得整个分子形成了一个大的硼掺杂的七环共 轭梯形平面结构. 在梯形分子中，两个嗍原子均为典型 的四面体构型. 七个环处于同一个平面上，而共轭体系 之外的两个米基基团与七环平面的夹角分别为 $89.14^{\circ}$ 和 $85.64^{\circ}$. 如此大的夹角表明两个米基都几乎垂直于共轭 平面, 成最大程度的伸展. 这样的结构可以有效地阻止 共轭平面之间的 $\pi-\pi$ 堆积，从而防止其聚集诱导荧光淬 灭, 使得梯形分子在固体状态下也有较强的苂光量子效 率. 这一点在右图的分子堆积结构图中也可以直观地看 到. 每一个分子的七环共轭平面都被平面上下的米基基 
团所阻断，这样使得分子平面之间没有 $\pi-\pi$ 相互作用， 分子和分子之间也没有分子间氢键和 $\mathrm{C}-\mathrm{H} \cdots \pi$ 等弱的 相互作用.

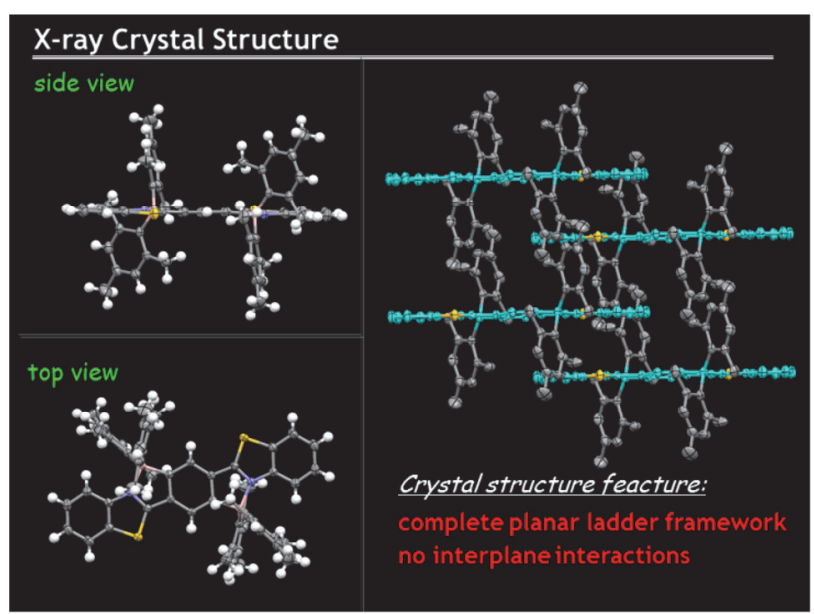

图 2 梯形嗍共轭分子的晶体结构图(左图为单分子结构图, 右图为分 子堆积结构图)

Figure 2 The crystal structure of the diboron ladder-type $\pi$-conjugated molecule (the left figure is single molecular structure and the right one is the packing structure)

\section{3 光物理性质}

为了研究梯形硼共轭分子的光物理性质, 我们分别 测试了其在二氯甲烷稀溶液中和薄膜状态下的紫外吸 收光谱和苂光发射光谱(图 3). 在溶液中, 化合物的最大 吸收波长位于 $372 \mathrm{~nm}$ 处, 根据之前的文献报道, 这个吸 收谱带应该归属于梯形共轭骨架的 $\pi-\pi$ *的跃迁. 化合物 的苂光发射峰位在 $544 \mathrm{~nm}$, 呈现绿光发射, 其苂光量子 效率为 0.17 . 可以得到, 化合物具有一个较大的斯托克 斯位移, 达到 $8499 \mathrm{~cm}^{-1}$. 由此推测: 该化合物的激发态 和基态相比, 具有较大的形变 ${ }^{[13]}$. 化合物的蒸镀薄膜发 射峰位在 $548 \mathrm{~nm}$ 处, 与溶液状态相比仅仅有很小的红

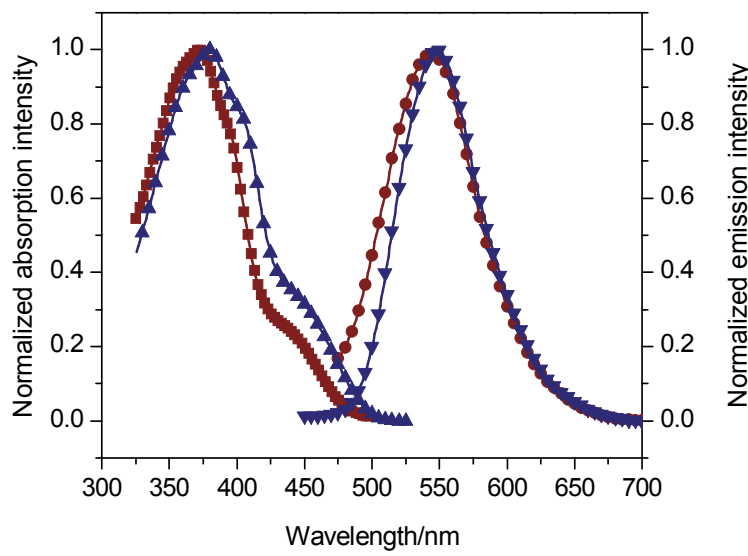

图 3 梯形嗍共轭分子在 $\mathrm{CH}_{2} \mathrm{Cl}_{2}$ 溶液 $\left(\left(1 \times 10^{-6} \mathrm{~mol} / \mathrm{L}\right.\right.$, 红线 $)$ 和真空蒸 镀薄膜(蓝线)的紫外可见吸收光谱和荧光发射光谱

Figure 3 The UV-vis absorption and fluorescence emission spectra in $\mathrm{CH}_{2} \mathrm{Cl}_{2}$ solution $\left(1 \times 10^{-6} \mathrm{~mol} / \mathrm{L}\right.$, red line $)$ and vacuum deposited thin film (blue line) of the diboron ladder-type $\pi$-conjugated molecule
移，并且化合物的荧光量子效率达到 0.15 . 与溶液相比, 几乎没有减弱. 这是由于米基基团的存在，极大地减弱 了分子之间的 $\pi-\pi$ 堆积，从而有效地避免了堆积引起的 苂光淬灭. 这样的光物理性质和分子堆积结构给出的信 息完全一致.

\section{4 热力学性质}

梯形硼共轭分子的热力学稳定性测试可以通过差 示扫描量热(DSC)和热重分析(TGA)来表征. 如图 4 所 示我们可以看到, 以每分钟 $10{ }^{\circ} \mathrm{C}$ 的升温速率对梯形硼 共轭分子的固体粉末进行第一轮加热的过程中，在 $352{ }^{\circ} \mathrm{C}$ 时有一个很明显的吸热峰. 冷却到室温之后, 再 以同样的速率进行第二循环的加热，与第一循环一样, 在同样的位置出现了吸热峰, 并且在其他位置没有任何 的吸热放热峰出现. 由此我们可以推断出, 这样一个梯 形硼共轭分子的熔点为 $352{ }^{\circ} \mathrm{C}\left(T_{\mathrm{m}}\right)$, 没有玻璃化转变 温度 $\left(T_{\mathrm{g}}\right)$ 和相转变温度 $\left(T_{\mathrm{c}}\right)$ 等. 也就是说: 这个分子具有 一个很高的熔点, 并且不存在玻璃态, 也没有其他的相 态之间的转变，表明该有机材料具有非常好的热稳定 性. 如图 4 内图所示, 对化合物的固体样品进行了热失 重分析测试. 同样地, 以每分钟 $10{ }^{\circ} \mathrm{C}$ 的升温速率对样 品进行加热, 我们发现当加热到 $360{ }^{\circ} \mathrm{C}$ 时, 样品损失的 质量达到了 $5 \%$. 所以说, $360{ }^{\circ} \mathrm{C}$ 对应于样品的热分解温 度 $\left(T_{\mathrm{d} 5}\right)$. 这在有机化合物中是一个很高的分解温度. 以 上测试都表明，我们所合成新型梯形硼共轭分子具有很 好的热稳定性. 这主要应该归因于化合物较大的分子量 以及这样一个非常刚性的梯形共轭平面. 众所周知, 好 的热稳定性可以使材料在加工过程中，尤其是在真空蒸 镀过程中, 避免不必要的结晶过程, 形成更加均一平整 的无定形膜. 好的无定形膜可以大大提高有机电致发光 器件(OLEDs) 的电致发光效率以及器件使用寿命等性 能.

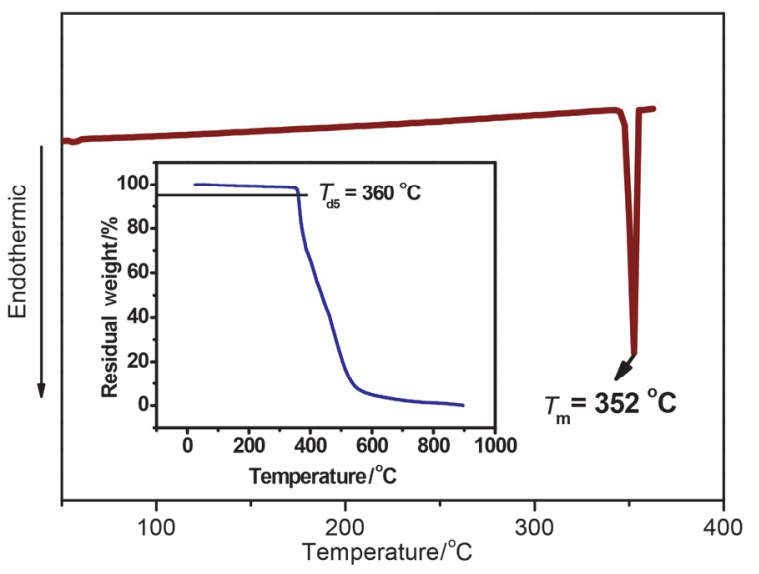

图 4 梯形嗍共轭分子的热力学稳定性测试(外图: 差示扫描量热 (DSC)曲线; 内图: 热失重分析(TGA)曲线)

Figure 4 The thermal stability of the diboron ladder-type $\pi$-conjugated molecule (outset: the DSC curve; inset: the TGA curve) 


\section{5 电化学性质}

梯形硼共轭分子的电化学性质通过循环伏安法 (CV)来进行测试. 氧化过程和还原过程测试分别在 1 $\mathrm{mmol} / \mathrm{L}$ 的 $\mathrm{CH}_{2} \mathrm{Cl}_{2}$ 和 THF 溶液中进行. 如图 5 所示: 化 合物表现出了可逆的还原峰和不可逆的氧化峰, 这与之 前报道的四配位嗍配合物是一致的. 在负向有两个完全 可逆的还原峰, 其半波电位分别为 -1.71 和 $-2.14 \mathrm{~V}$. 拥有两对可逆的还原峰的梯形嗍共轭分子也有文献报 道. 其中, 第一对可逆还原峰归属于梯形共轭骨架的还 原. 由此得到化合物的 LUMO 能级为 $-3.09 \mathrm{eV}$. 如此低 的 LUMO 能级是由于梯形双嗍的螯合作用导致的. 降 低 LUMO 能级可以使得阴离子的还原更加容易, 从而 提高配合物的电子亲和力. 在正向, 化合物的氧化过程 是不可逆的. 其氧化峰位 $\left(E_{\mathrm{oxp}}\right)$ 在 $+1.05 \mathrm{~V}$. 根据其氧化 峰的起始点, 可以得到其 HOMO 能级为 $-5.42 \mathrm{eV}$. 可 逆的还原电位和低的 LUMO 能级使得这样一个梯形路 共轭分子非常适合在有机电致发光发光器件(OLEDs)中 作为电子传输材料.

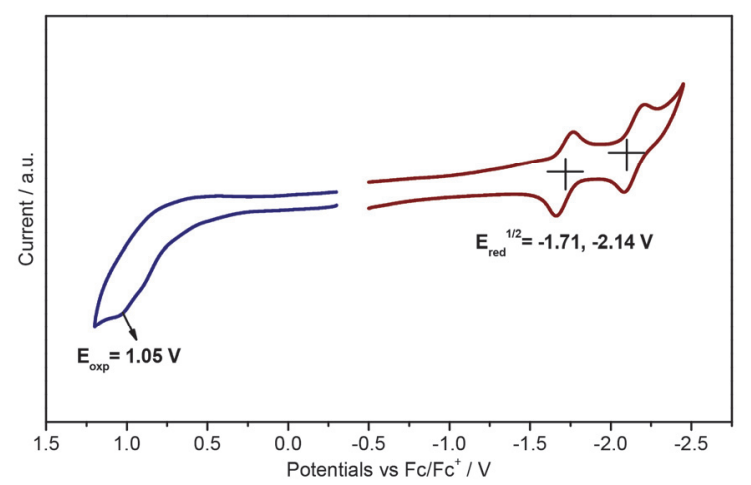

图 5 梯形硼共轭分子的循环伏安 $(\mathrm{CV})$ 曲线

Figure 5 Cyclic voltammogram curves of the diboron ladder-type $\pi$-conjugated molecule

\section{6 理论计算}

为了更加地深入理解梯形嗍共轭分子的电子结构, 我们利用密度泛函理论(DFT)对化合物进行了理论计算. 如图 6 所示, 化合物的最低非占有分子轨道(LUMO)几 乎完全由梯形并七环部分贡献, 而最高占有分子轨道 (HOMO)则主要由梯形结构中心的苯环以及与嗍螯合的 一个米基组成. 理论计算得到的 LUMO 能级值为 -2.58 $\mathrm{eV}, \mathrm{HOMO}$ 能级值为 $-5.36 \mathrm{eV}$. 可以看到理论计算和实 验得到的 HOMO 值差别不大, 而 LUMO 值相差比较大. 这可能是由于在配体螯合形成配合物的过程中, LUMO 能级被拉低了很多, 而由于这种基态密度泛函理论 (DFT)方法的局限性, 无法准确估算出这样的一个拉低 值. 虽然如此, 理论计算得到的轨道能级值与实验测得 的数值无论从变化趋势还是绝对值来说, 都已经非常接 近.

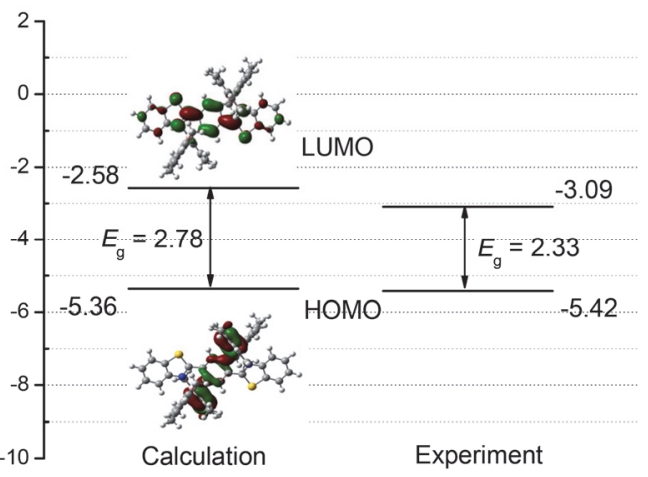

图 6 梯形硼共轭分子的理论计算的分子轨道分布图以及理论计算和 实验的最高占有分子轨道(HOMO)、最低非占有分子轨道(LUMO)能 级值对比

Figure 6 Calculated molecular orbital diagrams for HOMO and LUMO as well as calculated and experimental energy levels of the diboron ladder-type $\pi$-conjugated molecule

\section{7 电致发光性质}

考虑到化合物的梯形结构和以上优良的性质，我们 将其作为发光层和电子传输层分别构筑了电致发光器 件. 其单独作为发光层的器件结构为 [ITO/NPB (35 $\mathrm{nm}) / \mathrm{TCTA}(5 \mathrm{~nm}) / \mathbf{2}(30 \mathrm{~nm}) / \operatorname{Bepp}_{2}(30 \mathrm{~nm}) / \mathrm{LiF}(1 \mathrm{~nm}) / \mathrm{Al}$ $(200 \mathrm{~nm})$ ] (器件 D1). 其作为发光层和电子传输层的器 件结构为 $[\mathrm{ITO} / \mathrm{NPB}(35 \mathrm{~nm}) / \mathrm{TCTA}(5 \mathrm{~nm}) / 2(60 \mathrm{~nm}) / \mathrm{LiF}$ $(1 \mathrm{~nm}) / \mathrm{Al}(200 \mathrm{~nm})]$ (器件 D2). 其中, ITO(氧化铟锡)作 为阳极材料, NPB 作为空穴传输材料, TCTA 作为阻挡层 以阻止 NPB 与梯形硼化合物形成激基复合物, Bepp 2 作 为电子传输层 $\left(\mathrm{NPB} 、 \mathrm{TCTA} 、 \mathrm{Bepp}_{2}\right.$ 的结构式见支持信 息). 如图 7 所示, 器件的电致发光光谱与薄膜的光致发 光光谱很接近，表明：电致发光来源于梯形共轭分子的 本征发射, 并且空穴和电子的复合区域在梯形分子所在 的发光层. 梯形分子单独作为发光层的器件 D1 的开启 电压、最大亮度、最大电流效率和最大功率效率分别为 $3.0 \mathrm{~V}, 1476 \mathrm{~cd} / \mathrm{m}^{2}, 2.70 \mathrm{~cd} / \mathrm{A}, 2.82 \mathrm{~lm} / \mathrm{W}$. 为了考察梯形 分子的电子传输性质, 我们还构筑了器件 D2, 其开启 电压、最大亮度、最大电流效率和最大功率效率分别为 $3.2 \mathrm{~V}, 2415 \mathrm{~cd} / \mathrm{m}^{2}, 3.65 \mathrm{~cd} / \mathrm{A}, 3.58 \mathrm{~lm} / \mathrm{W}$. 与 D1 相比, 器 件 D2 在最大亮度和效率上都有提升. 表明, 梯形共轭 分子在发光和电子传输上, 都有比较好的应用潜能.

\section{3 结论}

我们利用一种简单的合成方法设计并合成了一个 新型双硼桥联的梯形分子. 单晶 X 射线衍射表明, 形成 的分子具有一个并七环结构的几乎共平面的 $\pi$ 共轭骨架. 这样的共轭骨架被空间位阻很大的米基取代基有效隔 开. 对其进行了光物理性质的研究, 由于该化合物特殊 的分子结构, 材料分子的聚集态荧光发射光谱较分散态 光谱只是略有红移, 并且两种状态下的苂光量子效率也 几乎相当. 热力学性质研究表明, 这样的梯形共轭分子 


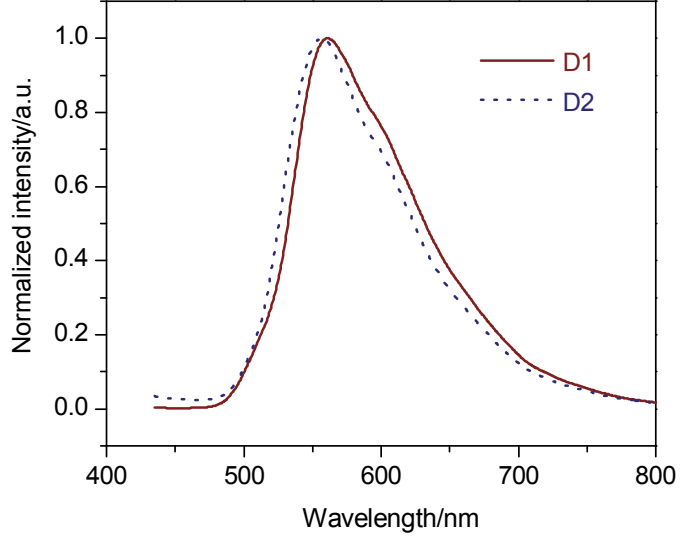

图 7 梯形硼共轭分子在 $100 \mathrm{~cd} / \mathrm{m}^{2}$ 亮度下的电致发光光谱 Figure 7 EL spectra of D1 and D2 at $100 \mathrm{~cd} \cdot \mathrm{m}^{-2}$

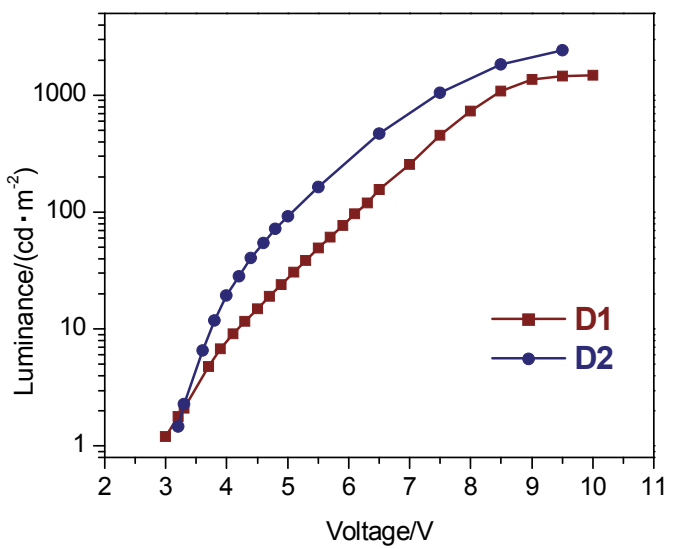

图 8 器件 D1 和 D2 发光亮度随电压的变化曲线

Figure 8 The luminance versus voltage curves of D1 and D2

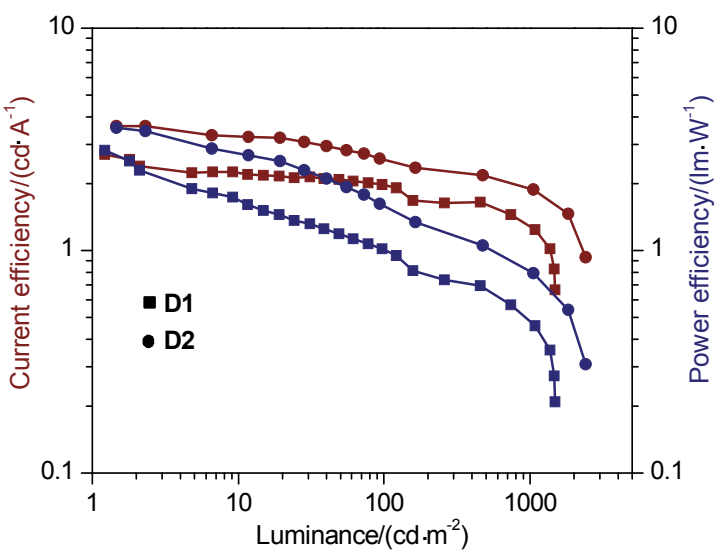

图 9 器件 D1 和 D2 电致发光效率随发光亮度的变化曲线

Figure 9 The efficiency versus luminance curves of D1 and D2

由于具有非常刚性的共轭结构能大大提高材料的热稳 定性. 电化学性质研究表明, 与之前报道的四配位梯形 嗍化合物类似, 其在负向有两对可逆的还原峰, 拥有较 低的 LUMO 能级, 具有很好的电子亲和力. 以上研究都 表明, 这样一个化合物在有机电致发光二极管器件中会
有很好的应用潜能. 基于此，我们构筑了该有机材料作 为发光材料和电子传输材料在有机电致发光二级管器 件, 取得了较好的器件效果.

\section{4 实验部分}

\section{1 化合物的合成}

在氮气保护下, 将 $3.24 \mathrm{~g}(10 \mathrm{mmol}) 2,5$-二溴对苯二 甲酸和 $3.02 \mathrm{~g}(24 \mathrm{mmol})$ 邻羟基苯硫酚加入到 $50 \mathrm{~mL}$ 多 聚磷酸中, $180{ }^{\circ} \mathrm{C}$ 下加热 $4 \mathrm{~h}$. 待溶液冷却至室温后, 倒 入水中进行水解. 待水解完全后, 抽滤. 将滤饼烘干得 到配体 1 的粗产品. 未经提纯直接加入到氮气保护的四 氢呋喃溶液中. $-78{ }^{\circ} \mathrm{C}$ 下滴加正丁基锂. 低温反应 $2 \mathrm{~h}$. 加入足量的二米基氟化硼, 缓慢恢复至室温, 搅拌过夜. 粗产品进行真空梯度升华, 得到 $1.26 \mathrm{~g}$ 橙黄色纯品, 两 步总产率 $15 \% .{ }^{1} \mathrm{H}$ NMR $\left(\mathrm{CDCl}_{3}, 500 \mathrm{MHz}\right) \delta: 8.27(\mathrm{~s}$, 2H), $7.95(\mathrm{~d}, J=10.0 \mathrm{~Hz}, 2 \mathrm{H}), 7.86 \sim 7.89(\mathrm{~m}, 2 \mathrm{H}), 7.38 \sim$ $7.45(\mathrm{~m}, 4 \mathrm{H}), 6.69(\mathrm{~s}, 8 \mathrm{H}), 2.20(\mathrm{~s}, 12 \mathrm{H}), 1.89(\mathrm{~s}, 24 \mathrm{H})$. Anal. calcd for $\mathrm{C}_{56} \mathrm{H}_{54} \mathrm{~B}_{2} \mathrm{~N}_{2} \mathrm{~S}_{2}$ : C 80.00, H 6.47, N 3.33, S 7.63; found C 79.88, H 6.51, N 3.45, S 7.46; MS $m / z$ : $840.1\left[\mathrm{M}^{+}\right]$(calcd 840.4).

\section{$4.2 X$ 射线单晶衍射}

单晶衍射数据的采集使用日本 Rigaku 公司生产的 R-AXIS RAPID X 射线单晶衍射仪, 在室温条件下, 用 石墨单色化的 Mo K $\alpha$ 射线 $(\lambda=0.71073 \AA)$, 以 $\omega$ 扫描方 式收集. 结构解析使用 SHELXTL-97 程序 ${ }^{[14]}$ 采用直接 法确定部分原子坐标位置, 进一步利用差傅里叶函数和 最小二乘法得到其他非氢原子的坐标位置, 所有非氢原 子都要进行各向异性精修; 然后通过理论加氢法确定所 有氢原子的坐标位置, 并使用最小二乘法对所求的结构 进行修正.

该梯形分子的分子式: $\mathrm{C}_{56} \mathrm{H}_{54} \mathrm{~B}_{2} \mathrm{~N}_{2} \mathrm{~S}_{2}$; 相对分子质 量 $\left(M_{\mathrm{r}}\right): 840.75$; 晶系: 单斜晶系; 晶胞参数: $a=9.891(2)$ $\AA, b=11.514(2) \AA, c=20.371(4) \AA, \alpha=90^{\circ}, \beta=90.59^{\circ}$, $\gamma=90^{\circ}$; 体积: $2319.8(8) \AA^{3}$; 密度实验值 $\left(D_{\mathrm{m}}\right): 1.204 \mathrm{~g} \cdot$ $\mathrm{cm}^{-3}$; 密度计算值 $\left(D_{\mathrm{c}}\right): 1.204 \mathrm{~g} \cdot \mathrm{cm}^{-3}$; 晶胞中分子数 $(Z)$ : 2; $F(000): 892.0$; 吸收系数 $(\mu): 0.155 \mathrm{~mm}^{-1} ; \mathrm{CCDC}$ 号: 1406572 .

\section{3 电化学测试}

电化学循环伏安法测试采用美国 BAS 公司生产的 $100 \mathrm{~W}$ 型电化学工作站. 电化学测量采用三电极体系: 铂电极作为工作电极, $\mathrm{Ag} / \mathrm{Ag}^{+}$电极作为参比电极, 铂丝 作为对电极. 在 $100 \mathrm{mV} \cdot \mathrm{s}^{-1}$ 扫速下进行测量, 二茂铁作 为内标, $0.1 \mathrm{~mol} / \mathrm{L}$ 的四丁基六氟磷酸铵的二氯甲烷和四 氢呋喃溶液作为支持电解质.

\section{4 理论计算}

理论计算采用 Gaussian 03 程序 ${ }^{[15]}$ 进行. 然后采用 密度泛函理论 DFT/B3LYP (Becke's three-parameter 
functional and the Lee-Yang-Parr functional)方法 ${ }^{[16]}$ 对研 究体系进行基态几何构型优化. 梯度优化中对 $N 、 C$ 和 $\mathrm{H}$ 原子使用价层䢃裂 $6-31 \mathrm{G}$ 和一个 $\mathrm{d}$ 极化函数基组 (6-31G(d)). 所有的计算都采用 Gaussian03 程序包.

\section{5 器件制备和测量}

用氧化铟锡(ITO)玻璃作为基底, 分别用丙酮、异丙 醇、去离子水清洗并超声. 然后对其进行等离子体处理. 器件在 $5 \times 10^{-5} \mathrm{~Pa}$ 真空度下制备. 有机物的蒸镀速度为 $0.3 \sim 0.5 \AA \cdot \mathrm{s}^{-1}$. 各层的蒸镀速率及厚度用石英晶振片来 监测. 器件的电流、电压、亮度、发光光谱等性质采用 PR650 亮度-光谱测试仪和 Keithley-2400 电流-电压测 试仪组成的系统进行测量. 所有测试均在室温大气中进 行.

\section{References}

[1] (a) Kawaguchi, K.; Nakano, K.; Nozaki, K. J. Org. Chem. 2007, 72, 5119. (b) Anthony, J. E. Chem. Rev. 2006, 106, 5028. (c) Bendikov, M.; Wudl, F.; Perepichka, D. F. Chem. Rev. 2004, 104, 4891. (d) Xiao, K.; Liu, Y.; Qi, T.; Zhang, W.; Wang, F.; Gao, J.; Qiu, W.; Ma, Y.; Cui, G.; Chen, S.; Zhan, X.; Yu, G.; Qin, J.; Hu, W.; Zhu, D. J. Am. Chem. Soc. 2005, 127, 13281. (e) Fukazawa, A.; Yamaguchi, S. Chemistry-An Asian Journal 2009, 4, 1386.

[2] (a) Liu, Z.-Q.; Fang, Q.; Wang, D.; Xue, G.; Cao, D.-X.; Yu, W.-T.; Xia, G.-M. Acta Chim. Sinica 2003, 61, 1449. (刘志强, 方奇, 王东, 薛刚, 曹笃霞, 于文涛, 夏光明, 化学学报, 2003, 61, 1449.) (b) Wang. W.; Fang, Q.; Liu, Z.-Q.; Cao, D.-X.; Deng, M.-Z. Acta Chim. Sinica 2005, 63, 1323. (王伟, 方奇, 刘志强, 曹笃霞, 邓敏 智, 化学学报, 2005, 63, 1415.) (c) Cao, D.-X.; Liu, Z.-Q.; Wang, D.; Fang, Q. Acta Chim. Sinica 2005, 63, 1415. (曹笃霞, 刘志强, 王东, 方奇, 化学学报, 2005, 63, 1415.) (d) Li, X.-H.; Xu, B.; Lv, K.-L.; Zhang, A.-Q. Chin. Chem. Lett. 2011, 22, 599.

[3] (a) Rao, Y.-L.; Wang, S. Inorg. Chem. 2011, 50, 12263. (b) Li, D.; Zhang, H.; Wang, Y. Chem. Soc. Rev. 2013, 42, 8416. (c) Zhang, H.; Huo, C.; Ye, K.; Zhang, P.; Tian, W.; Wang, Y. Inorg. Chem. 2006, 45, 2788. (d) Zhang, H.; Huo, C.; Zhang, J.; Zhang, P.; Tian, W.; Wang, Y. Chem. Commun. 2006, 281. (e) Zhang, Z.; Yao, D.; Zhao, S.; Gao, H.; Fan, Y.; Su, Z.; Zhang, H.; Wang, Y. Dalton Trans. 2010, 39, 5123. (f) Chen, H. Y.; Chi, Y.; Liu, C. S.; Yu, J. K.; Cheng, Y. M.; Chen, K. S.; Chou, P. T.; Peng, S. M.; Lee, G. H.; Carty, A. J.; Yeh, S. J.; Chen, C. T. Adv. Funct. Mater. 2005, 15, 567. (g) Liu, Q. D.; Mudadu, M. S.; Thummel, R.; Tao, Y.; Wang, S. Adv. Funct. Mater. 2005, 15, 143.

[4] (a) Kubo, Y.; Watanabe, K.; Nishiyabu, R.; Hata, R.; Murakami, A.; Shoda, T.; Ota, H. Org. Lett. 2011, 13, 4574. (b) Chen, J. J.; Conron, S. M.; Erwin, P.; Dimitriou, M.; McAlahney, K.; Thompson, M. E. ACS Appl. Mater. 2015, 7, 662. (c) Yoshii, R.; Yamane, H.; Nagai, A.; Tanaka, K.; Taka, H.; Kita, H.; Chujo, Y. Macromolecules 2014, 47, 2316. (d) Min, J.; Ameri, T.; Gresser, R.; Lorenz-Rothe, M.; Baran, D.; Troeger, A.; Sgobba, V.; Leo, K.; Riede, M.; Guldi, D. M.; Brabec, C. J. ACS Appl. Mater. 2013, 5, 5609.

[5] Wang, L.; Zhang, Z.; Cheng, X.; Ye, K.; Li, F.; Wang, Y.; Zhang, H. J. Mater. Chem. C 2015, 3, 499.

[6] (a) Boens, N.; Leen, V.; Dehaen, W. Chem. Soc. Rev. 2012, 41, 1130. (b) Madhu, S.; Ravikanth, M. Inorg. Chem. 2012, 51, 4285. (c) Lifschitz, A. M.; Shade, C. M.; Spokoyny, A. M.; Mendez-Arroyo, J.; Stern, C. L.; Sarjeant, A. A.; Mirkin, C. A. Inorg. Chem. 2013, 52, 5484. (d) Sarkar, S. K.; Mukherjee, S.; Thilagar, P. Inorg. Chem.
2014, 53, 2343. (e) Tian, M.-Z.; Feng, F.; Meng, S.-M.; Yuan, Y.-H. Chinese Chem. Lett. 2009, 20, 326.

[7] (a) Cui, Y.; Liu, Q.-D.; Bai, D.-R.; Jia, W.-L.; Tao, Y.; Wang, S. Inorg. Chem. 2005, 44, 601. (b) Zhang, Z.; Zhang, H.; Jiao, C.; Ye, K.; Zhang, H.; Zhang, J.; Wang, Y. Inorg. Chem. 2015, 54, 2652.

[8] (a) Liddle, B. J.; Silva, R. M.; Morin, T. J.; Macedo, F. P.; Shukla, R.; Lindeman, S. V.; Gardinier, J. R. J. Org. Chem. 2007, 72, 5637; (b) Xu, C.-Y.; Huang, K.-J.; Xie, W.-Z. Acta Chim. Sinica 2009, 67, 1075. (许春芗, 黄克靖, 谢宛珍, 化学学报, 2009, 67, 1075.) (c) He, L.-Y.; Hu, Q.-Z.; Xi, H.-T.; Sun, X.-Q. Chin. J. Org. Chem. 2015, 35, 232 (in Chinese). (贺琳彦, 胡全子, 席海涛, 孙小强, 有 机化学, 2015, 35, 232.) (d) He, Y.; Feng, R.-K.; Yi, Y.-R.; Liu, Z.-X. Chin. J. Org. Chem. 2014, 34, 2236. (何源, 冯若昆, 易云瑞, 刘占 祥, 有机化学, 2014, 34, 2236.) (e) Ye, J.-H.; Ye, W.-F.; Xiao, C.-T.; Chen, Y.; Wang, G.-P.; Zhang, W.-C. Chin. J. Org. Chem. 2012, 32, 1503. (叶家海, 叶文芳, 肖承涛, 陈雨, 王光普, 张文超, 有机化 学, 2012, 32, 1503.) (f) Lei, Y.-P.; Cao, S.-W. Chin. Chem. Lett. 2010, 21, 135.

[9] (a) Son, H.-J.; Han, W.-S.; Wee, K.-R.; Chun, J.-Y.; Choi, K.-B.; Han, S. J.; Kwon, S.-N.; Ko, J.; Lee, C.; Kang, S. O. Eur. J. Inorg. Chem. 2009, 2009, 1503. (b) Job, A.; Wakamiya, A.; Kehr, G.; Erker, G.; Yamaguchi, S. Org. Lett. 2010, 12, 5470. (c) Matsuo, K.; Saito, S.; Yamaguchi, S. J. Am. Chem. Soc. 2014, 136, 12580.

[10] (a) Li, D.; Zhang, Z.; Zhao, S.; Wang, Y.; Zhang, H. Dalton Trans. 2011, 40, 1279. (b) Li, D.; Wang, K.; Huang, S.; Qu, S.; Liu, X.; Zhu, Q.; Zhang, H.; Wang, Y. J. Mater. Chem. 2011, 21, 15298. (c) Li, D.; Yuan, Y.; Bi, H.; Yao, D.; Zhao, X.; Tian, W.; Wang, Y.; Zhang, H. Inorg. Chem. 2011, 50, 4825. (d) Zhang, Z.; Bi, H.; Zhang, Y.; Yao, D.; Gao, H.; Fan, Y.; Zhang, H.; Wang, Y.; Wang, Y.; Chen, Z.; Ma, D. Inorg. Chem. 2009, 48, 7230.

[11] (a) Iida, A.; Yamaguchi, S. J. Am. Chem. Soc. 2011, 133, 6952. (b) Fukazawa, A.; Yamaguchi, E.; Ito, E.; Yamada, H.; Wang, J.; Irle, S.; Yamaguchi, S. Organometallics 2011, 30, 3870.

[12] Curiel, D.; Más-Montoya, M.; Usea, L.; Espinosa, A.; Orenes, R. A.; Molina, P. Org. Lett. 2012, 14, 3360.

[13] (a) Yamaguchi, E.; Wang, C.; Fukazawa, A.; Taki, M.; Sato, Y.; Sasaki, T.; Ueda, M.; Sasaki, N.; Higashiyama, T.; Yamaguchi, S. Angew. Chem. Int. Ed. 2015, 54, 4539. (b) Zhang, D.; Wen, Y.; Xiao, Y.; Yu, G.; Liu, Y.; Qian, X. Chem. Commun. 2008, 4777. (c) Hu, R.; Gomez-Duran, C. F. A.; Lam, J. W. Y.; Belmonte-Vazquez, J. L.; Deng, C.; Chen, S.; Ye, R.; Pena-Cabrera, E.; Zhong, Y.; Wong, K. S.; Tang, B. Z. Chem. Commun. 2012, 48, 10099. (d) Mao, M.; Xiao, S.; Li, J.; Zou, Y.; Zhang, R.; Pan, J.; Dan, F.; Zou, K.; Yi, T. Tetrahedron 2012, 68, 5037.

[14] (a) SHELXTL, Version 5.1; Siemens Industrial Automation, Inc. 1997. (b) Sheldrick, G. M., SHELXS-97, Program for Crystal Structure Solution, University of Göttingen, Göttingen, 1997.

[15] Frisch, M. J.; Trucks, G. W.; Schlegel, H. B.; Scuseria, G. E.; Robb, M. A.; Cheeseman, J. R.; Montgomery, J. A.; Vreven, T.; Kudin, K. N.; Burant, J. C.; Millam, J. M.; Iyengar, S. S.; Tomasi, J.; Barone, V.; Mennucci, B.; Cossi, M.; Scalmani, G.; Rega, N.; Petersson, G. A.; Nakatsuji, H.; Hada, M.; Ehara, M.; Toyota, K.; Fukuda, R.; Hasegawa, J.; Ishida, M.; Nakajima, T.; Honda, Y.; Kitao, O.; Nakai, H.; Klene, M.; Li, X.; Knox, J. E.; Hratchian, H. P.; Cross, J. B.; Adamo, C.; Jaramillo, J.; Gomperts, R.; Stratmann, R. E.; Yazyev, O.; Austin, A. J.; Cammi, R.; Pomelli, C.; Ochterski, J. W.; Ayala, P. Y.; Morokuma, K.; Voth, G. A.; Salvador, P.; Dannenberg, J. J.; Zakrzewski, V. G.; Dapprich, S.; Daniels, A. D.; Strain, M. C.; Farkas, O.; Malick, D. K.; Rabuck, A. D.; Raghavachari, K.; Foresman, J. B.; Ortiz, J. V.; Cui, Q.; Baboul, A. G.; Clifford, S.; Cioslowski, J.; Stefanov, B. B.; Liu, G.; Liashenko, A.; Piskorz, P.; Komaromi, I.; Martin, R. L.; Fox, D. J.; Keith, T.; Al-Laham, M. A.; Peng, C. Y.; Nanayakkara, A.; Challacombe, M.; Gill, P. M. W.; Johnson, B.; Chen, W.; Wong, M. W.; Gonzalez, C.; Pople, J. A. Gaussian 03, Revision C.02, Gaussian, Inc., Pittsburgh, PA, 2003.

[16] Runge, E.; Gross, E. K. U. Phys. Rev. Lett. 1984, 52, 997. 